\title{
Clinical evidence does not support the use of adjuvant radiotherapy in pancreatic cancer
}

\author{
John P Neoptolemos
}

Adjuvant chemoradiation has demonstrated survival benefits compared with radiotherapy for the treatment of advanced pancreatic cancer; however, there is no evidence that chemoradiation is superior to maintenance chemotherapy. ${ }^{1,2}$ In both the advanced and adjuvant settings there is no evidence that chemoradiation combined with maintenance chemotherapy prolongs survival beyond that achieved with maintenance chemotherapy. ${ }^{3-5}$ Whilst most countries have adopted adjuvant chemotherapy rather than chemoradiotherapy, the adjuvant treatment approach is controversial in North America.

Two groups have assessed data that support the use of adjuvant therapy by performing a retrospective analysis of statistics from the SEER registry. 6,7 Both studies concluded that there was improved survival with use of adjuvant radiotherapy compared with resection alone. Notably, the results of the two studies contradict each other. Hazard et al. analyzed 3,008 patients treated during the period 1988-2002. The 1,267 $(42 \%)$ patients who received radiotherapy achieved a median survival of 17 months and a 5 -year overall survival rate of $13 \%$, compared with 12 months and $9.7 \%$, respectively, for patients who only had a resection. ${ }^{6}$ Radiotherapy improved cause-specific survival in patients who had regional-lymph-node involvement $\left(T_{1}\right.$ $N_{1}$ to $T_{3} N_{1}$ ) but not in patients without regional involvement $\left(T_{1} N_{0}\right.$ to $\left.T_{2} N_{0}\right)$ or in those with direct extension beyond the pancreas without lymph-node involvement $\left(\mathrm{T}_{3} \mathrm{~N}_{0}\right)$. Artinyan et al. assessed 1,930 patients with $\mathrm{N}_{0}$ disease treated within the period 1988 to 2003. The median survival was 20 months in irradiated patients compared with 15 months for nonirradiated patients. ${ }^{7}$ When patients who survived for longer than 3 months were excluded, there was no difference in survival between the two groups, but on multivariate analysis the positive effect of radiotherapy remained.

Opposing conclusions were drawn from these two studies regarding the efficacy of adjuvant
The

comparison

highlights

the potential

dangers in

retrospective

data analysis

and potential

investigator

bias.

JP Neoptolemos is

The Owen and Ellen

Evans Chair of Cancer

Studies and Head

of the Division of

Surgery and Oncology,

University

of Liverpool,

Liverpool, UK.

\section{Competing interests}

The author declared no

competing interests.

www.nature.com/clinicalpractice doi:10.1038/ncponc1188 radiotherapy with regard to lymph-node status, despite the fact that both studies assessed almost identical data sets. ${ }^{6,7}$ Key data missing from the SEER registry include performance status and resection margin involvement, both of which are major prognostic variables. This comparison highlights the potential dangers in retrospective data analysis and potential investigator bias. ${ }^{8}$ Only patients with good performance status can receive adjuvant treatment and will, therefore, inevitably survive longer as a group irrespective of the efficacy of the additional treatment.

From 1989 to 1995, Sener et al. assessed data from 100,313 patients from the National Cancer Data Base, 9,044 (9\%) of whom underwent a resection. ${ }^{9}$ The best 5-year overall survival rate was observed for patients who had resection only (23.4\%); patients treated with adjuvant irradiation had a survival rate of $13.7 \%$.

It is for the aforementioned reasons that randomized controlled trials are so valued in informing the evidence base. In the ESPAC-1 trial, median survival was 19.9 months and the 5 -year survival rate was $13.2 \%$ for patients randomized to adjuvant combination chemoradiation and maintenance chemotherapy, compared with 21.6 months and $29 \%$, respectively, for those treated with maintenance chemotherapy alone. ${ }^{3}$ The findings were supported by the results of the CONKO-01 trial, in which the median and overall survival rates were 22.1 months and $22.5 \%$, respectively, for patients randomized to adjuvant gemcitabine, compared with 20.2 months and $11.5 \%$ for those randomized to observation. ${ }^{10}$ The relative benefits of fluorouracil/folinic acid or gemcitabine will be addressed by the ESPAC-3 trial, which is expected to report in early 2009. Together, these results show support for the use of adjuvant chemotherapy but not adjuvant radiotherapy.

Supplementary information in the form of a reference list is available on the Nature Clinical Practice Oncology website. 